\title{
Madman im Digitalen. \\ Donald Trumps Rückgriff auf eine Figur des Kalten Krieges
}

\author{
Tobias Nanz
}

Ihren Schock angesichts der Wahl Donald Trumps zum 45. Präsidenten der Vereinigten Staaten von Amerika verarbeiteten viele politische Gegner*innen gerne mit der vermeintlichen Gewissheit, dass das Amt den Präsidenten formen und zähmen würde. Viele Vorgänger Trumps hätten im Wahlkampf markige Sprüche von sich gegeben, seien aber dann - unter Einwirkung der Bürokratie, des Beamt*innenapparates und der Zwänge des politischen Körpers - ruhiger und staatstragender geworden. Die Hoffnung, dass auch Trump im Regierungsalltag die Wahlkampfrhetorik ablegen und zur Mäßigung finden wird, scheint sich bis dato nicht zu erfüllen. Deshalb wurden bereits mit der Amtseinführung die Stimmen lauter, die die Vorzüge eines Impeachment-Verfahrens anpriesen und auf Richard Nixon als historische Referenz verwiesen: Nixon trat - nachhaltig geschädigt von der Watergate-Affäre und ihren Folgen - von seinem Amt zurück, als er realisieren musste, dass selbst republikanische Senator*innen seine Absetzung unterstützen würden. Sein Rücktritt kam der Amtsenthebung zuvor.

Weniger bekannt als die Watergate-Affäre ist die Madman-Strategie, deren Autorschaft Nixon einmal beiläufig beanspruchte und die er im Vietnamkrieg mit zweifelhaftem Erfolg anwandte. Entlang dieser Strategie inszeniert sich der männliche politische Führer als irrationaler Akteur und Entscheider, um in einer Konfliktsituation größtmöglichen Druck auf Gegner*innen aufzubauen. Mögliche irrationale Entscheidungen und Verhandlungen werden so $\mathrm{zu}$ einem Mittel der Abschreckungspolitik. Es kursieren Spekulationen, ob Trump ebenso ein Anhänger der Madman-Strategie sei. Dies wäre nicht die einzige Gemeinsamkeit der beiden Präsidenten: Trump steht wie Nixon damals in dem Ruf, die Presse und andere kritische Massenmedien $\mathrm{zu}$ verachten, und auch in seinem

T. Nanz $(\square)$

Darmstadt, Deutschland

E-Mail: nanz@ privacy-trust.tu-darmstadt.de 
Mitarbeiter*innenkreis findet sich stets jemand, der dem Staatswohl unverträgliche und impulsiv gegebene Anweisungen ignoriert oder vertrauliche Informationen preisgibt (für Nixon vgl. Haldeman 1978: 181-188; für Trump vgl. Woodward 2018: xix).

In diesem Aufsatz sollen die Gemeinsamkeiten der rationalen Irrationalität der beiden US-Präsidenten ausgelotet und zugleich die Brüche, die - so die These vor allem in den unterschiedlichen Medien und im Medienhandeln liegen, diskutiert werden. Der Madman Trump ist mit seinem digitalen Mediengebrauch ein vollkommen anderer Madman als Nixon, der seine Irrationalität über andere, nicht-öffentliche Kanäle verbreitete. Dafür wird in einem ersten Schritt die Genese der Madman-Strategie erkundet, um Nixons Praktiken zu befragen. Schließlich werden Trumps Madman-Handlungen im digitalen Raum erläutert.

\section{Abschreckung im Kalten Krieg}

Die Madman-Strategie ist keine kohärente Konzeption, die jemals in der Spielund Abschreckungstheorie des Kalten Krieges ausgearbeitet worden wäre. Als Idee taucht sie aber um 1960 an verschiedenen Stellen auf, insbesondere in der Schrift The Strategy of Conflict von Thomas Schelling, der sich als Ökonom Ende der 1950er und in der ersten Hälfte der 1960er Jahre mit den Strategien des Nuklearkrieges befasste. Die Spieltheorie, die Schelling in der zeitgenössischen Forschung vorfand, erschien ihm für seine Zwecke nicht praktikabel und wurde deshalb bis dato auch nicht auf Fragen der Abschreckung angewendet (Schelling 1960: 10). Die Spieltheorie überbetonte seiner Ansicht nach den reinen, reißbrettartigen Konflikt, dessen Wettbewerbscharakter die beteiligten Parteien nur unilateral handeln lässt und keinen Anreiz zur Kollaboration bietet. Zudem fußte die Spieltheorie auf einer mathematischen Grundlage, was dazu führte, dass die Lösung eines Konfliktes zur Symmetrie tendierte (Ayson 2004: 22). Schelling arbeitete nun daran, seinen theoretischen Ansatz mit der Spieltheorie zu verbinden.

Ein Baustein zur Entwicklung der Theory of Strategy ist die Bestimmung von rationalen und irrationalen Entscheidungen politischer oder militärischer Akteur*innen. Hier kann man bereits Schellings Kritik an unausgewogener, polarisierender Theoriebildung bemerken. Denn kein*e Entscheidungsträger*in, so Schelling, lasse sich einem definitiven Wert auf einer Skala zuordnen, die von vollkommen rationalen Entscheidungen bis hin zu vollkommen irrationalen Entscheidungen reiche. Im Gegenteil entscheiden Akteur*innen auf der Basis von Grundlagen, die sowohl rational wie auch irrational sein oder wirken können.

So mag rationales Handeln das Ergebnis eines konsistenten Wertesystems sein, das die Basis für die Kalkulation der nächsten Schritte bildet. Irrationales Handeln mag umgekehrt resultieren aus einem inkonsistenten Wertesystem, aus Fehlkalkulationen, aus einem Informationsdefizit oder aus gestörter Kommunikation (Schelling 1960: 16-18). Dabei können sich die Grenzen zwischen Ratio und 
Irratio schnell verunklaren und beobachtungsabhängig sein: Eine Entscheidung, die auf unvollständigen Informationen oder gestörter Kommunikation basiert, kann in diesem beschränkten Rahmen rational getroffen worden sein, nach außen hin aber auf eine*n Akteur*in, der/die über mehr Informationen verfügt, irrational wirken.

Dies muss nicht zwingend von Nachteil sein, sondern kann auch als ein strategischer Vorzug eingesetzt werden. So können - Schelling verweist hier auf die Erfahrung mit seinen eigenen Kindern - auf der Seite der bedrohten Partei gezieltes Weghören, ein vermeintliches Nicht-Verstehen oder ein gelegentlicher Kontrollverlust die Drohungen und die Abschreckung einer anderen Partei außer Kraft setzen. Was für das Handeln von Familien gilt, trifft seiner Meinung nach auch auf Staaten zu. Wird ein Staat von einem anderen bedroht oder erpresst, kann es von Vorteil sein, irrationales Verhalten an den Tag zu legen, sei es real oder sei es simuliert: Zweifel an der Selbstkontrolle, Störungen im Kommunikationssystem oder unzureichende Informationen können die Entschlossenheit einer Partei brechen, da diese sich nicht sicher sein kann, ob ihre Drohung oder Erpressung überhaupt verstanden wurde (Schelling 1960: 18). Denn Abschreckung kann nur dann als Methode funktionieren, wenn die Bedrohungslage von einer gegnerischen Partei vollständig erkannt wurde und so die Lage richtig eingeschätzt werden konnte, damit sie ihr künftiges Handeln danach ausrichten wird. Umgekehrt kann die Strategie auch seitens der drohenden Partei wirken. Wenn eine drohende Partei sich einen Ruf erworben hat, verrückt und unberechenbar zu sein, wird die bedrohte Partei in Krisen mehr Vorsicht walten lassen, da sie nicht vorhersehbare Reaktionen erwarten muss. So ist es für die Konfliktlösung durchaus sinnvoll, nicht immer dem Postulat der vollkommenen Rationalität zu folgen. ,,[I]t is not a universal advantage in situations of conflict to be inalienably and manifestly rational in decision and motivation“ (ebd.), schlussfolgerte Schelling in seiner Schrift.

Schelling ist nicht der einzige Abschreckungstheoretiker, der im Kalten Krieg die rationale Irrationalität diskutierte. Sein Kollege Herman Kahn, der wie zweitweise auch Schelling Mitarbeiter der Denkfabrik RAND-Corporation war, bemerkte in seinem Buch Thinking about the Unthinkable, dass die USA nicht nur den ,cautious and responsible Soviet decision maker“ abschrecken sollten, sondern dass „,even the mad“ oder ,,an irrational enemy“ (Kahn 1962: 111-112) von einem Angriff abgehalten werden müssten. Henry Kissinger, der mit Schelling an der Harvard University tätig war, bevor er ins Weiße Haus berufen wurde, diskutierte in einer Schrift irrationales Verhalten als politisches Mittel und wies auf die problematische Seite hin: „Given the values of our society, the feat of convincing the Soviet leaders of our capacity for irrationality and our own people of our devotion to peace is probably insuperable." (Kissinger 1961: 45) Madmen laufen Gefahr, den Rückhalt ihrer Wähler*innen zu verlieren.

Während der Wissenschaftler Schelling abstritt, eine Madman-Strategie entworfen zu haben, und gleichzeitig seine Abneigung gegenüber dem Einsatz einer solchen Taktik auf höchster Regierungsebene hervorhob (Dodge 2006: 146), 
erklärte sich der Politiker Nixon in einem Gespräch mit seinem Stabschef Harry Robbin Haldeman selbst zum Urheber der Madman-Strategie:

„I call it the Madman Theory, Bob [= Robbin]. I want the North Vietnamese to believe I've reached the point where I might do anything to stop the war. We'll just slip the word to them that, ,for God's sake, you know Nixon is obsessed about communism. We can't restrain him when he's angry - and he has his hand on the nuclear button " - and Ho Chi Minh himself will be in Paris in two days begging for peace" (Haldeman 1978: 83).

Die Essenz von Nixons Madman-Strategie bestand darin, einer gegnerischen Partei Furcht vor irrationalen, unvorhersehbaren und dabei äußerst aggressiven Handlungen einzuflößen. Dazu gehört auch die implizite Androhung eines Atomschlages (Burr/Kimball 2015: 83).

Vor dem Hintergrund des Vietnamkrieges war Nixon unzufrieden über die ausbleibenden Fortschritte in den Verhandlungen zur friedlichen Lösung des Krieges in Südostasien. Deshalb entschloss er sich in enger Abstimmung mit seinem nationalen Sicherheitsberater Henry Kissinger, Druck auf Nordvietnam und die Sowjetunion auszuüben, um eine bessere Verhandlungsposition bei den geheimen Friedensgesprächen in Paris zu erlangen. Dafür bedienten sich Kissinger und Nixon der good cop/bad cop-Taktik: Während Nixon als Präsident gezeichnet wurde, der den Kommunismus verachtet und zur Beendigung des Krieges größte militärische Gewalt anwenden würde, war Kissinger für Verhandlungen offen, die dem (vermeintlichen) Zorn Nixons zuvorzukommen könnten (ebd.: 86).

Für Vietnam bedeutete dies, dass der bad cop Nixon einerseits zur Abschreckung die US Air Force im Oktober in Alarmzustand versetzte, gar die höchste Stufe (dies ist umstritten) - DEFCON 1 - wählte, andererseits jede militärische Eskalation zu verhindern suchte (Hersh 1983: 124; zit. n. Sagan/ Suri 2003: 156). Verstärkte Flugmanöver und Patrouillen nahe dem sowjetischen Luftraum sowie die atomare Bewaffnung der Langstreckenbomber sollten vom Systemgegner bemerkt werden, wurden aber vor der Öffentlichkeit streng geheim gehalten. Der good cop intensivierte die Gesprächsdiplomatie: Im Vorfeld erklärte Kissinger seinen nordvietnamesischen Verhandlungspartnern in Paris, dass man die ,greatest consequences“ ins Auge fassen müsse, wenn die Gespräche bis zum 1. November keine signifikanten Fortschritte erzielen würden. Parallel zu den militärischen Aktionen erklärte Kissinger dem sowjetischen Außenminister Dobrynin, dass der Präsident über die bisherigen Gespräche enttäuscht und seine Geduld am Ende sei (Sagan/Suri 2003: 159 f.).

Obgleich Nixon hier einige Eigenschaften eines Madman exerziert - er scheint einen Atomkrieg zu riskieren und wird von seinen Mitarbeiter*innen als entschlossen und kompromisslos dargestellt -, ist die Bilanz seines Bluffs ernüchternd. Weder die Sowjetunion noch Nordvietnam ließen sich von den Drohungen wahrnehmbar einschüchtern. Dies lag vielleicht daran, dass die Manöver im erhöhten Alarmzustand eher als „carefully controlled exercises“ und weniger als Bedrohung wirkten, da Nixon und Kissinger auf stärkere Provokationen verzichteten, auch um Unfälle zu verhindern, die zu einer handfesten Krise hätten 
führen können (ebd.: 180). Zudem wurden Maßnahmen nicht durchgeführt, die bei einem atomaren Schlag standardmäßig durchgeführt worden wären: Viele AtomU-Boote verblieben in ihren Häfen und die Bomber wurden nicht auf zivile Flughäfen verteilt, wie es bei früheren Krisen üblich war (ebd.: 176). Schließlich war es der nordvietnamesischen Führung nicht entgangen, dass die US-amerikanischen Bürger*innen den Krieg nicht mehr unterstützten. Der Abzug der US-amerikanischen Truppen schien so nur noch eine Frage der Zeit zu sein, weshalb die nordvietnamesischen Vertreter ohnehin kein starkes Interesse an Verhandlungen hatten (Haldeman 1978: 98). Nixon musste einsehen, dass sein Bluff nicht glaubhaft war. „Incidentally“, so stellte Schelling das Madman-Konzept einmal klar, „that successful ,madman“ is not bluffing, unless he's only pretending to be mad" (Dodge 2006: 146).

\section{Madman Trump im digitalen Raum}

Seit der Wahl Trumps zum US-Präsidenten wurden Stimmen laut, die Vergleiche zwischen dem Reality Show-Star und Nixon zogen sowie die Madman-Strategie hinter Trumps Verhalten vermuteten. So wurde darauf verwiesen, dass er bereits im Wahlkampf die - seiner Ansicht nach - vorhersehbare Politik seiner Vorgänger kritisierte und eine weniger durchsichtige Außenpolitik forderte. Im April 2016 erklärte er: „We are totally predictable. We tell everything. We're sending troops? We tell them. We're sending something else? We have a news conference. We have to be unpredictable [...]“" (Nedal/Nexon 2017).

Wie ein vorbildlicher Schüler Nixons und Kissingers soll er im Handelsstreit mit Südkorea seinem Bevollmächtigten Robert Lighthizer folgende Handelsstrategie vorgegeben haben: Als Lighthizer den asiatischen Verbündeten eine dreiBigtägige Bedenkzeit einräumen wollte, unterbrach ihn Trump: ,That's not how you negotiate. You don't tell them they've got 30 days. You tell them, ,This guy's so crazy he could pull out any minute“" (Swan 2017). Diese Verhandlungsstrategie erscheint als Erprobungsfeld eines Madman im Amt und zielt zweifellos auf die Erhöhung des politischen Drucks zur Erpressung eines befreundeten und militärisch abhängigen Staates.

Ganz im Sinne Nixons verweisen Unterstützer*innen Trumps nun darauf, dass eine solche Unberechenbarkeit gegnerische Parteien abschrecken würde, während besorgte Beobachter*innen die Glaubwürdigkeit der Vereinigten Staaten, insbesondere gegenüber alliierten Staaten, in Gefahr sehen (Nedal/Nexon 2017). Andere fürchten, dass ein Madman Trump auf einen weiteren, vielleicht viel verrückteren Madman mit atomarer Bewaffnung treffen könnte (Slater 2018), sodass beide - um das Chicken Game aus der Spieltheorie zu zitieren - in Autos mit herausgerissenen Lenkrädern aufeinander zurasen, sodass niemand bluffen und ausweichen kann. Mit Blick auf die Drohungen gegen Nordkorea warnte der sich im Ruhestand befindliche General David Petraeus, dass die Madman-Strategie zwar ein durchaus legitimes Mittel sei, aber in der Katastrophe enden könnte: Wenn die Führung eines bedrohten Staates von Trumps Irrationalität überzeugt sei, 
könnte jene sich entschließen, einen Präventivschlag gegen die USA auszuführen, was bei einer Atommacht in die Apokalypse führe (Berke 2017).

Mit Blick auf die internationale Handelspolitik glauben andere Beobachter*innen wiederum, dass Trump Nixon nicht richtig verstanden hätte: Nixon spielte zwar den Madman, um Druck auszuüben, hätte sich aber am Verhandlungstisch vernünftig und klar verhalten; bei Trump könne man den zweiten Schritt, die rationale Seite des Madman, bislang nicht erkennen (Levy 2018).

Politisch und theoretisch ist der Madman im Kalten Krieg verwurzelt. Trotz seines zweifelhaften Erfolges scheint er auch heute noch einen strategischen Platz in der Trump-Politik einzunehmen. Wenn man sein Auftreten, seine Verhandlungen und Entscheidungen hinsichtlich dieser Strategie befragen will, muss man die veränderte geopolitische Lage wie auch die Medienumbrüche - hier insbesondere die Digitalisierung - in die Analysen miteinbeziehen.

Während im Kalten Krieg das bipolare System die dominante weltpolitische Konzeption war, die allerdings stets von schwächeren und stärkeren Dritten (Reichherzer/Droit/Hansen 2018) wie China, Europa und etwa blockfreien Staaten unterlaufen wurde, rückten mit dem Zerfall der Sowjetunion, mit dem Aufkommen von sog. ,Schurkenstaaten ' sowie mit dem Krieg gegen den Terror neue Bedrohungen in den Fokus. Aus der dominanten bipolaren Weltordnung wurde eine undurchsichtige Welt mit vielen Krisenherden, für die die simulierten Panzerschlachten des Kalten Krieges keinen Maßstab mehr darstellen. Die globale Sicherheitslage im frühen 21. Jahrhundert ist geprägt von Cyberwar, Drohnen und Netzwerkkriegen. Atomwaffen erscheinen hier schon fast anachronistisch, obgleich diese ein gewichtiges Pfund in den Händen eines Madman sind.

Im Jahr 2017 stießen zwei Madmen mehrfach aufeinander. Nachdem Nordkorea am 3. Juli, einem Tag vor dem Unabhängigkeitstag der USA, den ersten Test einer Langstreckenrakete durchführte, twitterte Trump wütend: „Does this guy have anything better to do with his life?" (03. Juli 2017, 09:19:02) ${ }^{1}$ Am 8. August, nach weiteren Raketentests, folgte die mediale Eskalation: Am Rande einer Kabinettssitzung erklärte der Präsident der Presse und damit der Öffentlichkeit, dass Nordkorea bei fortgesetzten Drohungen die nukleare Auslöschung erwarte: „They will be met with fire and fury like the world has never seen“", so Trumps berühmte Warnung via Fernsehen (NBC 2017). Die nordkoreanische Führung erwiderte, die nukleare Vernichtung der US-amerikanischen Pazifikinsel Guam in Erwägung zu ziehen. Dies provozierte Trump erneut zu einem Twitter-Vorstoß, der an einen Showdown im Western-Film erinnert: „Military solutions are now fully in place, locked and loaded, should North Korea act unwisely. Hopefully Kim Jong Un will find another path!“ (11. August 2017, 06:29:31). Dieser Nachricht folgte ein Retweet einer Botschaft des US Pacific Command, die einen Trainingsflug eines B1-B Lancer-Bombers ankündigt, der von Guam in Richtung koreanischer Halbinsel führe (11. August 2017, 08:19:02).

\footnotetext{
${ }^{1}$ Die folgenden Tweets wurden im Trump Twitter Archive recherchiert.
} 
Nach einer kurzzeitigen Beruhigung der Lage griff Trump den Konflikt bei seiner ersten Rede vor der UN-Vollversammlung am 19. September wieder auf. „Rocket man“, so wie er Kim Jong-un invektiv adressierte, ,is on a suicide mission for himself. [...] The United States has great strength and patience. But if it is forced to defend itself or its allies we will have no choice but to totally destroy North Korea" (CNN 2017).

Die Antwort Nordkoreas ließ nicht lange auf sich warten: Der US-amerikanische Präsident sei „mentally deranged“ sowie ,unfit to hold the prerogative of supreme command of a country“. Zudem, so hieß es, erwäge man einen erbarmungslosen Gegenschlag (Jong-un 2017). Trump forderte nun seinen Kontrahenten, Madman gegen Madman, öffentlich zu einem Chicken Game heraus: „Kim Jong Un of North Korea, who is obviously a madman who doesn't mind starving or killing his people, will be tested like never before!"“ (22. September 2017, 05:28:02).

Glücklicherweise wurde dieses Spiel nie realisiert, doch Trump twitterte weiter über „Little Rocket Man“ und die Unmöglichkeit, mit diesem zu verhandeln. So schrieb er Anfang Oktober: „I told Rex Tillerson, our wonderful Secretary of State, that he is wasting his time trying to negotiate with Little Rocket Man..." (01. Oktober 2017, 09:30:59) Wenige Stunden später führte er seinen Tweet fort: „Being nice to Rocket Man hasn't worked in 25 years, why would it work now? Clinton failed, Bush failed, and Obama failed. I won't fail“" (01. Oktober 2017, 14:01:19). In diesen Tweets ist indes unklar, ob Trump nun seinen Minister oder seinen Widersacher mit größerer Ironie belegt. In jedem Fall wurde Kim Jong-un zum innenpolitischen Instrument, mit dem der Präsident seinen Außenminister entmachtete und sich gleichzeitig als den entschlossensten Präsidenten der jüngeren Geschichte inszenierte. Der öffentliche Verzicht auf die Kompetenz des Außenministeriums rückte in diesem Zuge den Atomknopf näher an den Madman im Oval Office heran.

Ausgehend von dieser Konfrontation erscheinen nun vier Aspekte für die Charakterisierung eines Madman im digitalen Zeitalter charakteristisch: Erstens erweist sich Twitter, Trumps bevorzugter Social Media Messenger, als besonders geeignet, um den US-amerikanischen Präsidenten als Madman zu zeichnen. Die maximale Länge einer Botschaft beträgt 280 Zeichen, sodass größere Reflexionen, ein Abwägen oder das Vorbringen eines komplexeren Arguments gar nicht möglich sind - auch wenn Trump gelegentlich eine Botschaft in mehreren Tweets verschickt. Twitter, ursprünglich als ein Dienst gegründet, um ,pointless babble“ (Rogers 2014: xiii) zu pflegen, also mitzuteilen, ob man gerade etwas isst oder bereits zu Bett gegangen ist, hat sich von dieser Anfangsphase emanzipiert, tendiert aber nach wie vor zu emotionaler, griffiger, aber unterkomplexer Kommunikation. Bei Trump wurde gar in einer empirischen Studie festgestellt, dass er zu einem hohen Prozentsatz wütende Nachrichten versendet, die sich gar nicht auf einen bestimmten Fall beziehen, sondern allgemeinen Ärger transportieren (Wahl-Jorgensen 2018: 82). Ein Madman, der wüten und drohen möchte, wird in Twitter sein ideales Medium finden. 
Zweitens suggeriert Twitter eine vermeintliche Unmittelbarkeit zwischen dem Sender Trump und seinen Millionen Followers. Die emotionale Botschaft erreicht unter Auslassung der zum Staatsfeind erklärten Massenmedien direkt die Empfänger*innen (Delli Carpini 2018: 20), ohne - so wird zumindest suggeriert - von jemandem aus Trumps Team gegengelesen zu werden. Eine Reihe Tweets erzeugt einen Zustand der Dauererregung, einer „ekstatischen Exklamatorik“ (Strohschneider 2018: 66), die aus einem schriftlichen Text eine Ansprache macht, die mündlicher Rede ähnelt und eine distanzlose Unmittelbarkeit vermittelt. Die Tweets simulieren eine unverfängliche, freundschaftliche Konversation mit seinen Anhänger*innen (Turner 2018: 148). So scheinen diese direkten Zugang zu ihm zu haben, der, wie ein zentrales Versprechen des Populismus lautet, weiß, was das Volk will, das sonderbarerweise mit einer Stimme zu sprechen vermag (Müller 2016: 44).

Drittens erreicht Trump mit seinen Tweets eine außerordentlich große Öffentlichkeit globalen Ausmaßes, die seine Statements beim Wort nehmen kann. Während Nixon sich davor scheute, seine camouflierten Drohungen gegenüber Vietnam und der Sowjetunion öffentlich zu machen und jede bemerkbare Truppenverlegung vermied, verkündet Trump seine Beleidigungen und möglichen Aktionen einem Millionenpublikum. Verstärkt werden seine Tweets zudem nicht nur durch Retweets, sondern auch durch die Zitation und Diskussion der Inhalte in den klassischen Massenmedien. Trumps Tiraden kann man nicht entkommen, weder in den digitalen sozialen Medien noch in den von ihm so verhassten älteren Massenmedien.

Viertens erwarb sich Trump seit Beginn seiner Amtszeit einen Ruf als notorischer Lügner (siehe die Dokumentation der Washington Post: Anonym 2019), der Fakten ignoriert und die Massenmedien als Staatsfeinde behandelt, die vermeintlich allenthalben Fake News verbreiten. So errichtet er sich eine Welt, in der in erster Linie eine Anschlusskommunikation nur mit seinen Anhänger*innen möglich ist, eine Hyperrealität (Baudrillard 1978; Delli Carpini 2018: 19) also, die sich in ihrer medialen Formation mithilfe von sozialen Netzwerken sowie in der Verbreitung von Lügen via Massenmedien von den vertrauten politischen Praktiken und Diskursen der westlichen Nachkriegs- und Kalten Kriegs-Gesellschaft abhebt. Die Nachricht von den neuen Sternen, also jenem hyperrealen TrumpUniversum, kündet von einem zweifelhaften Aufbruch in eine neue politische und gesellschaftliche Welt und mag von verwundertem Augenreiben oder zynischen Kommentaren begleitet werden.

Trump ist so - fasst man diese Aspekte zusammen - einerseits ein Madman, der alle Möglichkeiten der digitalen Netzwerke zur Selbstinszenierung und Gemeinschaftsbildung $\mathrm{zu}$ nutzen sucht, dort irrationales sowie emotionales Handeln ausstellt und einer großen Öffentlichkeit bekanntmacht. Damit lassen sich offensichtlich seine Wähler*innen begeistern sowie von seinen Dealmaker-Qualitäten überzeugen. Andererseits ist er kein Madman, jedenfalls keiner im politischen und strategischen Sinne, da aufgrund seiner Negierung von Fakten das Vertrauen schwindet, dass der Madman nach den Androhungen verrückter Aktionen und dem 
folgenden Einlenken der Bedrohten dazu in der Lage ist, seriöse und rechtsverbindliche Verhandlungen zu führen. Denn ein Madman lässt sich nicht einseitig mit irrationalem Handeln charakterisieren, sondern muss - um politische Wirkung zu erzeugen - in der Lage sein, nach der Ankündigung irrationaler Aktionen rationale und diplomatisch verbindliche Entscheidung zu treffen. Auch sollte sein Bluff glaubhaft sein, sodass gegnerische Parteien die Einlösung der Drohungen für möglich halten. Allerdings erscheint die Lücke zwischen der Hyperrealität Trumps und der Lebenswirklichkeit zu groß.

Es bleibt die Sorge, dass keine Medien mehr zwischen beiden Sphären vermitteln können. In der einen, hyperrealen Welt findet das Wüten und Schimpfen eines alten, weißen Mannes statt, das aus Überzeugung und Voyeurismus begeistert geteilt oder entschieden abgelehnt wird. In der anderen Welt, in der man das „Ende der Diplomatie“ (Farrow 2018) betrauert, wird man erkennen müssen, dass die alte Diplomatie nie so durchgehend rational und besonnen war, wie sie im Kontrast zu Trumps Politik erscheint. Im Gegenteil ist Trump in einer Hinsicht noch sehr in der alten Welt verhaftet.

Denn seine Inszenierungen weisen gleichermaßen wie der Wahlspruch „Make America Great Again“ auf die Zeit des Kalten Krieges, dessen bipolare Strategien nichts mehr mit der multipolaren Welt des 21. Jahrhunderts gemein haben. Der Madman Kahns, Kissingers, Nixons oder Schellings, jene Figur, die aus den ungeheureren Forschungs- und Rüstungsanstrengungen des Kalten Krieges hervorgegangen ist und dabei nie sonderlich erfolgreich war, ist als politische sowie militärstrategische Option verschwunden, als mit dem Ende des Kalten Krieges ebenso die Spieltheorie in der Politik ihre Relevanz verlor (Erickson u. a. 2013: 183-184). Von der rationalen Irrationalität scheint bei Trump nur noch ein Rest, nämliche die (gespielte) Verrücktheit übriggeblieben zu sein. Der atemberaubende Kurswechsel von atomaren Vernichtungsdrohungen Nordkoreas hin zu Freundschaftsbekundungen - „my friend Kim“ - (26. Februar 2019, 09:31:21), der Schwenk von der Erzeugung größtmöglicher Furcht hin zu dem Versprechen einer großen Zukunft auf der koreanischen Halbinsel - ,[Kim's] country could fast become one of the great economic powers" (24. Februar 2019, 08:19:19) lässt aus Politik eine Angelegenheit der Laune und der populistischen Reden werden, bei der die rationale Perspektive nur schwer erkennbar ist. Der Madman des Kalten Krieges irrlichtert durch das 21. Jahrhundert.

\section{Literatur}

Anonym: In 801 days, President Trump has made 9,451 false or misleading claims. The Fact Checker's ongoing database of the false or misleading claims made by President Trump since assuming office. In: The Washington Post (31.03.2019), https://www.washingtonpost.com/ graphics/politics/trump-claims-database/?utm_term=.f80dd2b96ad8 (11.04.2019).

Ayson, Robert: Thomas Schelling and the Nuclear Age. Strategy as Social Sciences. London/New York 2004.

Baudrillard, Jean: Agonie des Realen. Berlin 1978. 
Berke, Jeremy: Petraeus says Trump's ,madman' approach to North Korea could be effective until it becomes disastrous. In: Business Insider Deutschland (16.09.2017), https://www. businessinsider.de/david-petraeus-on-trumps-madman-approach-to-north-korea-kim-jong-un2017-9? $\mathrm{r}=\mathrm{US} \& \mathrm{IR}=\mathrm{T}$ (11.04.2019).

Burr, William/Kimball, Jeffrey P.: Nixon's Nuclear Specter. The Secret Alert of 1969, Madman Diplomacy, and the Vietnam War. Lawrence 2015.

CNN: Trump to UN: ,Rocket Man' on a suicide mission (19.09.2017), https://www.youtube. $\mathrm{com} / \mathrm{watch} ? \mathrm{v}=8 \mathrm{mstcJDHaGE}(11.04 .2019)$.

Delli Carpini, Michael X.: Alternative facts. Donald Trump and the emergence of a new U.S. media regime. In: Pablo J. Boczkowski/Zizi Papacharissi (Hg.): Trump and the Media. Cambridge, Mass./London 2018: 17-23.

Dodge, Robert: The Strategist. The Life and Times of Thomas Schelling. Hollis 2006.

Erickson, Paul/Klein, Judy L./Daston, Lorraine/Lemov, Rebecca/Sturm, Thomas/Gordin, Michael D.: How Reason almost lost its Mind. The Strange Career of Cold War Rationality. Chicago/London 2013.

Farrow, Ronan: Das Ende der Diplomatie. Warum der Wandel der Amerikanischen Außenpolitik für die Welt so gefährlich ist. Reinbek bei Hamburg 2018.

Haldeman, Harry R.: The Ends of Power. London 1978.

Hersh, Seymour M.: The Price of Power. Kissinger in the Nixon White House. New York 1983.

Jong-un, Kim: Full text of Kim Jong-un's response to president Trump. In: The New York Times (22.09.2017), https://www.nytimes.com/2017/09/22/world/asia/kim-jong-un-trump. html (11.04.2019).

Kahn, Herman: Thinking about the Unthinkable. New York 1962.

Kissinger, Henry A.: The Necessity for Choice. Prospects of American Foreign Policy. New York 1961.

Levy, Phil: Trump and the half-madman theory of international negotiations. In: Forbes (25.04.2018), https://www.forbes.com/sites/phillevy/2018/04/25/trump-and-the-half-madmantheory-of-international-negotiations/\#13e8000f7461 (11.04.2019).

Müller, Jan-Werner: Was ist Populismus? Ein Essay. Berlin 2016.

NBC News: Donald Trump: North Korea , will be met with fire and fury“ (08.08.2017), youtube. com, https://www.youtube.com/watch?v=1bt4t05m_j0 (11.04.2019).

Nedal, Dani/Nexon, Daniel: Trumps ,Madman Theory“ isn't strategic unpredictability. It's just crazy. In: Foreign Policy (18.04.2017), https://foreignpolicy.com/2017/04/18/trumps-madman-theory-isnt-strategic-unpredictability-its-just-crazy/ (11.04.2019).

Reichherzer, Frank/Droit, Emmanuel/Hansen, Jan (Hg.): Den Kalten Krieg vermessen. Über Reichweite und alternativen einer binären Ordnungsvorstellung. Berlin/Boston 2018.

Rogers, Richard: Foreword: Debanalising twitter: The transformation of an object of study. In: Kartin Weller/Axel Bruns/Jean Burgess/Merja Mahrt/Cornelius Puschmann (Hg.): Twitter and Society. New York 2014: ix-xxvi.

Sagan, Scott D./Suri, Jeremi: The madman nuclear alert. Secrecy, signaling, and safety in october 1969. In: International Security 27/4 (2003): 150-183.

Schelling, Thomas C.: The Strategy of Conflict. Cambridge, Mass./London 1960.

Slater, Jerome: Trump is using the ,madman theory' in North Korea policy. In: The National Interest (03.06.2018), https://nationalinterest.org/feature/trump-using-the-madman-theorynorth-korea-policy-26097 (11.04.2019).

Strohschneider, Peter: POTUS als Twitterer. In: Zeitschrift für Ideengeschichte 12/3 (2018): $61-75$.

Swan, Johnatan: Trump urges staff to portray him as „crazy guy“. In: Axios (01.10.2017), https://www.axios.com/scoop-trump-urges-staff-to-portray-him-as-crazy-guy-1513305888c1cbdb89-6370-4e13-98ed-28c414e62a35.html (11.04.2019).

Trump Twitter Archive, http://www.trumptwitterarchive.com (11.04.2019). 
Turner, Fred: Trump on twitter. How a medium designed for democracy became an authoritarian's mouthpiece. In: Pablo J. Boczkowski/Zizi Papacharissi (Hg.): Trump and the Media. Cambridge, Mass./London 2018: 143-149.

Wahl-Jorgensen, Karin: Public displays of disaffection: The emotional politics of Donald Trump. In: Pablo J. Boczkowski/Zizi Papacharissi (Hg.): Trump and the Media. Cambridge, Mass./ London 2018: 79-86.

Woodward, Bob: Fear. Trump in the White House. London 2018.

Open Access Dieses Kapitel wird unter der Creative Commons Namensnennung 4.0 International Lizenz (http://creativecommons.org/licenses/by/4.0/deed.de) veröffentlicht, welche die Nutzung, Vervielfältigung, Bearbeitung, Verbreitung und Wiedergabe in jeglichem Medium und Format erlaubt, sofern Sie den/die ursprünglichen Autor(en) und die Quelle ordnungsgemäß nennen, einen Link zur Creative Commons Lizenz beifügen und angeben, ob Änderungen vorgenommen wurden.

Die in diesem Kapitel enthaltenen Bilder und sonstiges Drittmaterial unterliegen ebenfalls der genannten Creative Commons Lizenz, sofern sich aus der Abbildungslegende nichts anderes ergibt. Sofern das betreffende Material nicht unter der genannten Creative Commons Lizenz steht und die betreffende Handlung nicht nach gesetzlichen Vorschriften erlaubt ist, ist für die oben aufgeführten Weiterverwendungen des Materials die Einwilligung des jeweiligen Rechteinhabers einzuholen. 